\title{
Sperm Retrieval Gold Standard Predictors: TXNDC2, PRM1 and PRM2, Joint Molecular Markers Associated With Testis Pathology
}

seyed-Morteza Javadirad ( $\sim$ javadirad@yahoo.com )

University of Isfahan

Mohammad Mokhtari

Tarbiat Modares University

\section{Research Article}

Keywords: Azoospermia, TXNDC2, PRM1, PRM2, Sperm retrieval

Posted Date: March 15th, 2021

DOI: https://doi.org/10.21203/rs.3.rs-283914/v1

License: (c) (i) This work is licensed under a Creative Commons Attribution 4.0 International License.

Read Full License 


\section{Abstract}

The necessity of PRM1/2 in male azoospermia has been approved, but the association of TXNDC2 deficiency with the phenotype, sperm retrieval and pathology has not been approved. Here we identified a joint cooperation of TXNDC2 and protamines in evaluating testis pathology and sperm retrieval. Extensive meta-analysis on human arrays of idiopathic, untreated and with unknown cause of azoospermia was done. After several steps of data quality controls, QC passed data were pooled and batch effect corrected. As Redox imbalance has been shown as a two edge sword toward fertility, wet lab studies began with candidated protamination and thioredoxin genes. Logistic regression model of TXNDC2 alongside PRM1 and PRM2 genes was built and collective ROC analysis indicated a sensitivity of $96.8 \%$ and specificity of $95.5 \%$ with the ROC value of 0.993 (SE=0.0075, 95\% Cl: $0.978-1.000)$. In conclusion, TXNDC2, PRM1 and PRM2 in joint, have a robust power to predict sperm retrieval and to correlate with severe pathology of azoospermia.

\section{Introduction}

Pathology uses the terms obstructive (OA) and non-obstructive azoospermia (NOA) respectively for normal and abnormal spermatogenesis. Pathology also classifies aberrant spermatogenesis into five main patterns ${ }^{1}$ : seminiferous tubules hyalinezation (SH), Sertoli cell-only syndrome (SCOS), early $\backslash$ late maturation arrest (e\IMA), and hypospermatogenesis (Hypo). We must note that testicular imperfection and seminiferous tubules obstruction status are the ultimate achievement of pathological analysis; however, the prediction of sperm retrieval (SR) could not be concluded solely based on the current approach.

Budget expenses and loss of golden time are two critics to treat azoospermic men, wishing to have their own biological children. Trustworthy, precise molecular markers with strong background toward spermatogenesis could be a booty for the heartbroken parents. To reduce the stress of infertile couples and to create a spark of hope especially for NOA men, we previously introduced KDM3A to PRM1 expression ratio as a reliable molecular indicator of $\mathrm{SR}^{2}$. But we could not spectacle any association between aforementioned genes and the pathological features of the biopsies. By scouting gene(s) with dual propensities we can kill two birds with one stone, predicting the success of SR, and also confirming testicular pathology. Having this bonus in our pocket, we can first join pathology and genetics to double check for SR possibility and foremost, surgeons could be persuaded to explore NOA tissues to extract any residual sperms at the first round of surgery.

Thioredoxines are intracellular and extracellular scavengers of oxidative stress system, reactive oxygen species (ROS) being one of their main targets, regulation of redox signaling plays pivotal roles in sperm fertility ${ }^{3}$. Thioredoxin domain containing 2 (TXNDC2, ENSG00000168454), is transiently expressed in haploid phase of spermatogenesis and as a sperm-specific oxidoreductase, is only detected in round and elongating spermatids ${ }^{45}$. TXNDC2/TXNDC3 double inactivation in animal models was performed and impaired chromatin protamination was the output ${ }^{6}$. Protamination as the DNA-safeguard, not only 
condensates sperm chromatin, but also replaces most of the histones during spermiogenesis and male infertility is conclusively associated with impaired protamination ${ }^{7}$. As we know, protamination is started by the expression of transition protein 1 (TNP1) and is followed by protamines (PRM1 and PRM2) replacement in the nucleus ${ }^{8}$; Thereafter, mature spermatozoa would be released into the lumen of seminiferous tubules $^{9}$. So it seems that protamines and sperm-specific thioredoxins are all together important for spermatozoa to start capacitation, bringing these proteins so necessary for male fertility ${ }^{10}$.

In this study, TXNDC3 was not evaluated as it is ubiquitously expressed in all tissues and is no longer considered testis specific ${ }^{11}$. Considering the localization of $T X N D C 2$ in the nucleus versus extracellular distribution of $T X N D C 8$, the latter was also removed. Therefore, the aim of this study was to evaluate the expression levels of $T X N D C 2$ together with protamination genes in different pathological status of azoospermia. We showed that PRM1 and PRM2, but not TNP1, are excellent indicators of SR. We also showed that $T X N D C 2$ expression levels were in complete agreement with the status of tissue pathologies. Moreover, logistic regression model analysis of $T X N D C 2, P R M 1$ and $P R M 2$ genes together was a robust predictor of SR by sensitivity of $96.8 \%$ and specificity of $95.5 \%$.

\section{Materials And Methods}

\section{Patients and samples}

Azoospermic men were double questioned before and after the operation and samples were ruled out after the operation, if they were not willing to continue their participation in the study. An approximately $50 \mathrm{mg}$ of fresh testicular tissues, were submerged immediately under the RNAlater stabilizing reagent (Ambion Life Science, Austin, TE, USA, AM7024) according to the manufacturer instruction. Micro-TESE open surgery team was fully informed as the first piece of testicular tissue was used for RNA extraction and the next pieces for pathology and SR. Submerged samples were stored at $4{ }^{\circ} \mathrm{C}$ for $24 \mathrm{~h}$ and then processed for further RNA extraction. Out of 50 samples included in, 40 were diagnosed as nonobstructive and 10 as obstructive-control individuals according to the pathological results. Inclusion and exclusion criteria were as follows: samples with weak RNA integrity, the ones with variable Cqs even after multiple rounds of separate analysis, and without clear pathology were omitted from this study. Unfortunately, we omitted 9 samples as they were reported with unknown pathology.

\section{Ethics statement}

Written informed consents were taken and full explanation was donated to azoospermic men before sampling. The experimentations and consent forms were approved by institutional review board of Isfahan University ethical committee. All procedures performed in the study involving human participants were in accordance with the 1964 Helsinki declaration and its later amendments or comparable ethical standards. 


\section{SR technique}

Schlegel technique was adopted and an expert surgeon has done all the micro-TESE open surgeries under the microscope in order to lessen the obstruction of testicular vessels ${ }^{12}$. Meticulous sperm processing with initial mechanical dissection of seminiferous tubules was followed by extensive exercise to receive the maximum rate of retrieval ${ }^{13}$.

\section{Histological analysis}

Hematoxylin and eosin (H\&E) staining of paraffin embedded tissues was performed according to the standard protoco ${ }^{14}$. Two microscopic slides containing at least 100 different tubular sections for each specimen were examined by a specialist pathologist and the results were reported as follows: (i) N: normal spermatogenesis with all types of spermatogenic cell lineages in sections, (ii) SH: seminiferous tubule hyalinization, (iii) SCOS: sertoli cell-only syndrome or germ cell aplasia, (iv) eMA: early maturation arrest, (v) IMA: late maturation arrest, (vi) Hypo: hypospermatogenesis. Individuals with normal spermatogenesis were considered as obstructive azoospermia (OA) and these were used as control individuals according to the previous reports ${ }^{15}$. Other pathologies with abnormal spermatogenesis were classified as non-obstructive azoospermia (NOA).

\section{GEO Meta-Analysis}

GEO database was explored with the keyword "azoospermia" for microarray datasets. A rigid inclusionexclusion criteria was applied as follow and a total of 9 datasets corresponding to homo sapiens species were found. Among these datasets those with any treatments and therapies were excluded. Samples with cryptorchidism phenotype and with detected mutations were also excluded. In this regard, GSE145467, GSE45885, GSE9194, GSE108886, GSE9210, GSE14310 were selected. All the candidated datasets were log2 scaled and quantile normalized if necessary. Hierarchical clustering of each dataset was illustrated using Euclidian distance. Principal component analysis (PCA) plot were drawn and outliers were detected and removed. GSE9194 and GSE9210 were excluded, respectively due to low quality and low feature intersection with other datasets. SVA ${ }^{16}$ and Limma ${ }^{17}$ packages were used to remove batch effects and subsequently, PCA and hierarchical clustering were used again to check the quality of the batch effect removal. Effect size of features was calculated using Limma package with Benjamini-Hochberg correction. We applied $p$ values to determine the corresponding false discovery rates (FDR). Finally, the variation of testis-specific thioredoxin gene2 (TXNDC2) alongside protamination genes (TNP1, PRM1, PRM2) were recorded. Testis-specific thioredoxin gene 8 (TXNDC8) was not included in GSE14310 dataset, and meta-analysis was performed on the resting GSE45885 and GSE108886 datasets. R version 4.0.1 was used for meta-analysis. 


\section{RNA isolation and cDNA synthesis}

RNA extraction was carried out as reported previously ${ }^{2}$. Nanodrop One (Thermo Scientific, USA) was used for quantification and then, $1 \mu \mathrm{g}$ of total RNA was treated with Dnasel (Thermo Scientific, Lithuania; EN0522) according to the manufacturer instruction. TaKaRa PrimerScript II $1^{\text {st }}$ stand cDNA synthesis kit (TaKaRa, Otsu, Japan; 6210B) was used to random prime the first strand of cDNA. The qualities of the extracted RNAs were checked by $2 \%$ conventional agarose gel electrophoresis stained with ethidium bromide (data not shown).

\section{Reverse transcription quantitative real-time PCR (RT-qPCR)}

Primers were adopted for RT-qPCR and their concentration was optimized according to our previous study. SYBR Premix Ex Taq II (TaKaRa; RR820L) was the quantifying dye in Corbett 6000 Rotor-Gene thermocycler (Corbett Life Science, Mortlake, Australia). Equal amounts of cDNA were amplified in triplicates and the average cycle of quantification $(\mathrm{Cq})$ values were further analyzed.

\section{Melting curve analysis}

After the last run of amplification, melting curve analysis via green channel was performed according to the manual of Corbett 6000 Rotor-Gene machine. Gradual increase in temperature $\left(1.0^{\circ} \mathrm{C} / \mathrm{s}\right)$ was applied from 65 to 95 degrees Celsius and the amount of fluorescence emission was recorded continuously. The deviation of fluorescence change over temperature on $y$ axis was plotted against the temperature on $x$ axis using Rotor-Gene embedded software 1.7.

\section{Gene expression analysis}

GAPDH and RPL37 were used simultaneously as reference genes for RT-qPCR data normalization based on our previous finding ${ }^{2}$. REST2009 was used for statistical analysis.

\section{Statistical analysis}

Raw mean Cqs were exported to SPSS v.21.0 (IBM Corp., Armonk, NY, USA) and normalization of the data was carried out if necessary. Normalized mean Cqs of the genes were compared between individuals with positive and negative SR using $t$-Test. A one-way between subjects ANOVA-coupled with Scheffe Post hoc comparison was conducted to visualize the differences of mRNA expression levels between different pathological status. Multiple linear regression approaches were applied to model the relationship between the expression levels of $P R M 1, P R M 2$, and $T X N D C 2$. A receiver operating characteristic curve (ROC) predictive model was obtained to demonstrate the predictive ability of the three expressed genes 
for SR. The area under the curve (AUC) was determined to assess the diagnostic accuracy. In all statistics, $p$ values smaller than 0.05 were considered as significant.

\section{Results}

\section{Data quality control and pre-processing:}

The assessment of data normalization revealed that parts of the data was log2 scaled, the resting were transformed and the second round of quality control was carried out to assess the quality of samples quantiles (supplementary Fig. 1). For each dataset, hierarchical cluster analysis of samples, based on Euclidian Distance of Pearson correlation coefficient, grouped similar objects into clusters. It was followed by the process of dimension reduction using the Eigen vector with the highest Eigen value (supplementary Fig. 2). The decision to remove 18 outliers out of 99 samples were based on sophisticated knowledge of biology, in combination with clustering and PCA (supplementary Fig. 3). Consequently, a total of 71 samples were pooled for further analysis.

Limma and SVA algorithms were applied on the pool to correct their batch. Then after, hierarchical clustering and Principle component analysis were performed, and the outcome brought us the confidence about the correction (Fig. 1).

\section{Meta-Analysis}

The gene expression of pooled data with pathological phenotypes of SCOS, pre-meiotic arrest, meiotic arrest, and post-meiotic arrest were evaluated (Fig. 2). Based on the goal of this study, protamination genes (PRM1, PRM2, TNP1) with respect to testis-specific thioredoxin genes (TXNDC2, TXNDC8) were analyzed and the data were presented in Table I and visualized in figures 3. SCOS patients' meta-analysis revealed meaningful downregulation of $T X N D C 2$ (effect size=-2.42, FDR=7.86E-07), $P R M 1$ (effect size = -4.28, FDR=5.89E-07), PRM2 (effect size = -3.98, FDR=1.77E-06), and TNP1 (effect size $=-4.75$, $\mathrm{FDR}=8.32 \mathrm{E}-09)$. Similar meaningful downregulation of the genes was also recorded in pre-meiotic arrest and meiotic arrest phenotypes, a situation failed to be confirmed in post-meiotic arrest. TXNDC2 (effect size $=-4.25, \mathrm{FDR}=1.44 \mathrm{E}-15), P R M 1$ (effect size $=-5.37, \mathrm{FDR}=1.99 \mathrm{E}-10), P R M 2$ (effect size $=-5.16$, FDR=3.60E-10), and TNP1 (effect size $=-7.05, F D R=6.48 E-16$ ) were all downregulated in idiopathic azoospermia dataset. Except for post-meiotic arrest, TXNDC8 meaningful downregulation was recorded for SCOS (effect size $=-1.59, F D R=3.97 E-05)$, pre-meiotic arrest (effect size $=-1.79$, FDR=8.63E-05) and meiotic arrest (effect size $=-1.55, \mathrm{FDR}=4.53 \mathrm{E}-05)$.

\section{RT-qPCR data analysis}

The mean expression level of GAPDH, RPL37, TXNDC2, PRM1, PRM2, and TNP1 were compared between positive and negative SR (sup. table I). Reference genes GAPDH and RPL37 showed the minimal mean 
differences between positive and negative $S R$ individuals $(0.59,0.97$ respectively). Considering positive SR as the control, high positive mean differences were recorded for TXNDC2, PRM1 and PRM2; but TNP1 showed a negative (-1.52) mean difference. Therefore, TXNDC2 was differentially expressed and in homology, protamination genes, PRM1 and PRM2. Unexpectedly, the expression of TNP1 was overlapping (Fig. 4). To test the significance of the observed differences, $t$-Test was performed on normalized data (Tablell). A significant differential expression for TXNDC2, PRM1, and PRM2 $(p=0.000)$ were observed between positive and negative SR, but not for TNP1 ( $p=0.558)$.

REST2009 relative expression analysis results were presented in Table III. Data analysis showed TXNDC2 significant downregulation with the expression ratio of $0.047(p=0.000)$. PRM1 and PRM2 genes were also significantly $(p=0.000)$ downregulated with the expression ration of 0.000 . TNP1, on the other hand, was insignificantly $(p=0.301)$ upregulated with slight expression ratio of 4.078 .

\section{Discussion}

Uninterrupted research interests toward introducing a suitable molecular marker to predict SR is a hot topic among researchers in the field of andrology. In the first attempt between different phenotype of azoospermia, only SCOS was successfully correlated with $R B M Y 1$, and $D A Z$ genes and they suggested a significant positive association between these genes and successful $\mathrm{SR}^{18}$. BOII/ GAPDH mRNA ratio was assessed in different pathological phenotypes of azoospermia, using the cut-off value of 0.5 , sensitivity and specificity of $100 \%$ was achieved for SR ${ }^{19}$.

Technical improvements made the methodology of the mentioned studies challenging and therefore, demands have risen for accurate and precise methods with the ability to diminish the biases. In accompany with this urgency, RT-qPCR was introduced and applied by numerus upcoming researches. ESX1 was the first introduced reliable molecular marker of spermatogenesis with significant $(p=0.04)$ concordance of $73.7 \%{ }^{20}$. Their further test of seminal fluid also confirmed the capacity of ESX1 as a molecular marker of SR with $84 \%$ sensitivity; albeit they declared discrepancies between molecular and clinical outputs ${ }^{15}$. In fulfilment of the previous studies, we improved the sensitivity of SR up to $95.5 \%$ using KDM3A histone demethylase; However, we were also unable to bring concordance between our molecular markers and pathological phenotypes ${ }^{2}$.

In the present study, TXNDC2 was correlated with SH phenotype, while PRM1 and PRM2 showed additional association with GCA/SCOS (Table IV). It is worth noting that genome wide integration of transcriptomics and anti-body based proteomics declared TXNDC8 as a testis specific protein as well, albeit as an extracellular equivalent of nuclear $T X N D C 2^{2111}$. It seems logical to consider $T X N D C 2$ over $T X N D C 8$, as protamine activation takes place in the nucleus. Furthermore, the association of $P R M 2$ but not $P R M 1$ with eMA was also interesting. In other words, these three genes could be altered at the very early stages of spermatogenesis; while when being expressed, it could be the sign of the existence of germ cells. As we know, protamine activation takes place before their DNA binding, the role that could be proposed for thioredoxin. After the release of protamine precursors, a round of sequential 
phosphorylation and dephosphorylation takes place to strengthen the binding power of protamines to wrap around the corresponding DNA. A key event after dephosphorylation, to complete the activation process, is the oxidation of protamine monomers to produce a head to tail dimer. Thioredoxins are oxidizing molecules acting on Cys residues, which are abundantly present in protamins. Therefore, synchronous downregulation of TXNDC2 and PRM1/PRM2 in SH and SCOS (the most severe phenotypes of sperm failure) could infer their importance for sperm production.

To future examine the observed synchronicity, linear regression model analysis was developed (Table V). TXNDC2 showed a strong correlation with both PRM1 $(\mathrm{r}=0.761)$ and PRM2 ( $\mathrm{r}=0.767)$. The coefficient of determination, correlates up to $60 \%$ of $P R M 1$ and $P R M 2$ expression solely with $T X N D C 2$ expression. Moreover, $P R M 1$ was perfectly correlated $(\mathrm{r}=0.993, p=0.000)$ with $P R M 2$; which means, the value of $P R M 2$ could be anticipated from $P R M 1$ by $98.6 \%$. Mentioned observations proposed similar behaviors of two co-expressed protamines toward each other. In accompany, animal knockout models and our previous study, confirmed KDM3A as the transcription factor of PRM1 and PRM2, itself under the control of HIF1$a^{28}$. It is also proved that the overexpression of thioredoxin could increase HIF1-a activity ${ }^{22}$.

To determine the clinical importance of our potential biomarkers of SR (TXNDC2 alongside PRM1 and $P R M 2)$, logistic regression model of the genes was built based on intact Cqs. Receiver Operator Characteristic (ROC) analysis was conducted afterward, to evaluate the predictive power of these 3 candidate biomarkers. Collective ROC analysis indicated a sensitivity of $96.8 \%$ and specificity of $95.5 \%$ with the ROC value (AUC) of 0.993(SE=0.0075, 95\% Cl: 0.978-1.000) (Fig. 5).

\section{Conclusion}

Taken together, it was cleared that TXNDC2 was differentially expressed between positive and negative SR. Moreover, TXNDC2 was correlated with severe phenotypes of azoospermia (SH and SCOS). A strong correlation of TXNDC2 with protamination genes was observed. ROC analysis applied on the multiple regression model of granted TXNDC2-PRM1-PRM2 as robust molecular markers of SR with sensitivity of $96.8 \%$ and specificity of $95.5 \%$.

\section{Declarations}

\section{Data availability}

The dataset (GSE145467, GSE45885, GSE9194, GSE108886, GSE9210, GSE14310) analyzed during the current study is available in the NCBI-Gene Expression Omnibus repository.

\section{Acknowledgements}

Authors sincerely thank the volunteers for their participation. 


\section{Author's roles}

Seyed-Morteza: Conception, design, assembly of data, data analysis, interpretation, financial supports, drafting the manuscript, revising it critically for important intellectual content, and final approval of the manuscript.

Mohammad: Conception, design, collection, and/or assembly of data, data analysis, interpretation, and drafting of the manuscript.

\section{Funding}

This study has been conducted in Isfahan University of Iran and was supported financially by the Departments of Research, Technology and Graduate Offices.

\section{Conflict of interest}

None of the authors has any conflicts of interest to disclose and all authors support submission to this journal.

\section{References}

1. Dohle GR, Elzanaty S and van Casteren NJ: Testicular biopsy: clinical practice and interpretation. Asian J. Androl. 2012; 14: 88-93.

2. Javadirad SM, Hojati Z, Ghaedi K, et al: Expression ratio of histone demethylase KDM3A to protamine-1 mRNA is predictive of successful testicular sperm extraction in men with obstructive and non-obstructive azoospermia. Andrology 2016; 4: 492-499. Available at: https://onlinelibrary.wiley.com/doi/abs/10.1111/andr.12164.

3. O'Flaherty C: Peroxiredoxins: hidden players in the antioxidant defence of human spermatozoa. Basic Clin. Androl. 2014; 24: 4. Available at: https://doi.org/10.1186/2051-4190-24-4.

4. Miranda-Vizuete A, Ljung J, Damdimopoulos AE, et al: Characterization of Sptrx, a novel member of the thioredoxin family specifically expressed in human spermatozoa. J. Biol. Chem. 2001; 276: 31567-31574.

5. Jiménez A, Johansson C, Ljung J, et al: Human spermatid-specific thioredoxin-1 (Sptrx-1) is a twodomain protein with oxidizing activity. FEBS Lett. 2002; 530: 79-84. Available at: https://doi.org/10.1016/S0014-5793(02)03417-8.

6. Smith TB, Baker MA, Connaughton HS, et al: Functional deletion of Txndc2 and Txndc3 increases the susceptibility of spermatozoa to age-related oxidative stress. Free Radic. Biol. Med. 2013; 65: 872881. 
7. Ni K, Spiess A-N, Schuppe H-C, et al: The impact of sperm protamine deficiency and sperm DNA damage on human male fertility: a systematic review and meta-analysis. Andrology 2016; 4: 789799. Available at: https://doi.org/10.1111/andr.12216.

8. Okada Y, Scott G, Ray MK, et al: Histone demethylase JHDM2A is critical for Tnp1 and Prm1 transcription and spermatogenesis. Nature 2007; 450: 119-123.

9. Lüke L, Campbell P, Varea Sánchez M, et al: Sexual selection on protamine and transition nuclear protein expression in mouse species. Proceedings. Biol. Sci. 2014; 281: 20133359. Available at: https://pubmed.ncbi.nlm.nih.gov/24671975.

10. Aitken RJ: The Capacitation-Apoptosis Highway: Oxysterols and Mammalian Sperm Function. Biol. Reprod. 2011; 85: 9-12. Available at: https://doi.org/10.1095/biolreprod.111.092528.

11. Fagerberg L, Hallström BM, Oksvold P, et al: Analysis of the Human Tissue-specific Expression by Genome-wide Integration of Transcriptomics and Antibody-based Proteomics * . Mol. Cell. Proteomics 2014; 13: 397-406. Available at: https://doi.org/10.1074/mcp.M113.035600.

12. Schlegel PN: Testicular sperm extraction: microdissection improves sperm yield with minimal tissue excision. Hum. Reprod. 1999; 14: 131-135.

13. Dabaja AA and Schlegel PN: Microdissection testicular sperm extraction: an update. Asian J. Androl. 2013; 15: 35-39.

14. Fischer AH, Jacobson KA, Rose J, et al: Hematoxylin and eosin staining of tissue and cell sections. CSH Protoc. 2008; 2008: pdb.prot4986.

15. Pansa A, Sirchia SM, Melis S, et al: ESX1 mRNA expression in seminal fluid is an indicator of residual spermatogenesis in non-obstructive azoospermic men. Hum. Reprod. 2014; 29: 2620-2627.

16. Leek JT, Johnson WE, Parker HS, et al: The sva package for removing batch effects and other unwanted variation in high-throughput experiments. Bioinformatics 2012; 28: 882-883.

17. Ritchie ME, Phipson B, Wu D, et al: limma powers differential expression analyses for RNAsequencing and microarray studies. Nucleic Acids Res. 2015; 43: e47-e47. Available at: https://doi.org/10.1093/nar/gkv007.

18. Kuo P-L, Wang S-T, Lin Y-M, et al: Expression profiles of the DAZ gene family in human testis with and without spermatogenic failure. Fertil. Steril. 2004; 81: 1034-1040.

19. Lin YM, Kuo PL, Lin YH, et al: Messenger RNA transcripts of the meiotic regulator BOULE in the testis of azoospermic men and their application in predicting the success of sperm retrieval. Hum. Reprod. 2005; 20: 782-788.

20. Bonaparte $\mathrm{E}$, Moretti M, Colpi GM, et al: ESX1 gene expression as a robust marker of residual spermatogenesis in azoospermic men. Hum. Reprod. 2010; 25: 1398-1403.

21. Balhorn R: The protamine family of sperm nuclear proteins. Genome Biol. 2007; 8: 227. Available at: https://doi.org/10.1186/gb-2007-8-9-227.

22. Naranjo-Suarez S, Carlson BA, Tobe R, et al: Regulation of HIF-1a activity by overexpression of thioredoxin is independent of thioredoxin reductase status. Mol. Cells 2013; 36: 151-157. 
23. Dohle GR, Elzanaty $S$ and van Casteren NJ: Testicular biopsy: clinical practice and interpretation. Asian J. Androl. 2012; 14: 88-93.

24. Javadirad SM, Hojati Z, Ghaedi K, et al: Expression ratio of histone demethylase KDM3A to protamine-1 mRNA is predictive of successful testicular sperm extraction in men with obstructive and non-obstructive azoospermia. Andrology 2016; 4: 492-499. Available at: https://onlinelibrary.wiley.com/doi/abs/10.1111/andr.12164.

25. O'Flaherty C: Peroxiredoxins: hidden players in the antioxidant defence of human spermatozoa. Basic Clin. Androl. 2014; 24: 4. Available at: https://doi.org/10.1186/2051-4190-24-4.

26. Miranda-Vizuete A, Ljung J, Damdimopoulos AE, et al: Characterization of Sptrx, a novel member of the thioredoxin family specifically expressed in human spermatozoa. J. Biol. Chem. 2001; 276: 31567-31574.

27. Jiménez A, Johansson C, Ljung J, et al: Human spermatid-specific thioredoxin-1 (Sptrx-1) is a twodomain protein with oxidizing activity. FEBS Lett. 2002; 530: 79-84. Available at: https://doi.org/10.1016/S0014-5793(02)03417-8.

28. Smith TB, Baker MA, Connaughton HS, et al: Functional deletion of Txndc2 and Txndc3 increases the susceptibility of spermatozoa to age-related oxidative stress. Free Radic. Biol. Med. 2013; 65: 872881.

29. Ni K, Spiess A-N, Schuppe H-C, et al: The impact of sperm protamine deficiency and sperm DNA damage on human male fertility: a systematic review and meta-analysis. Andrology 2016; 4: 789799. Available at: https://doi.org/10.1111/andr.12216.

30. Okada Y, Scott G, Ray MK, et al: Histone demethylase JHDM2A is critical for Tnp1 and Prm1 transcription and spermatogenesis. Nature 2007; 450: 119-123.

31. Lüke L, Campbell P, Varea Sánchez M, et al: Sexual selection on protamine and transition nuclear protein expression in mouse species. Proceedings. Biol. Sci. 2014; 281: 20133359. Available at: https://pubmed.ncbi.nlm.nih.gov/24671975.

32. Aitken RJ: The Capacitation-Apoptosis Highway: Oxysterols and Mammalian Sperm Function. Biol. Reprod. 2011; 85: 9-12. Available at: https://doi.org/10.1095/biolreprod.111.092528.

33. Fagerberg L, Hallström BM, Oksvold P, et al: Analysis of the Human Tissue-specific Expression by Genome-wide Integration of Transcriptomics and Antibody-based Proteomics * . Mol. Cell. Proteomics 2014; 13: 397-406. Available at: https://doi.org/10.1074/mcp.M113.035600.

34. Schlegel PN: Testicular sperm extraction: microdissection improves sperm yield with minimal tissue excision. Hum. Reprod. 1999; 14: 131-135.

35. Dabaja AA and Schlegel PN: Microdissection testicular sperm extraction: an update. Asian J. Androl. 2013; 15: 35-39.

36. Fischer AH, Jacobson KA, Rose J, et al: Hematoxylin and eosin staining of tissue and cell sections. CSH Protoc. 2008; 2008: pdb.prot4986.

37. Pansa A, Sirchia SM, Melis S, et al: ESX1 mRNA expression in seminal fluid is an indicator of residual spermatogenesis in non-obstructive azoospermic men. Hum. Reprod. 2014; 29: 2620-2627. 
38. Leek JT, Johnson WE, Parker HS, et al: The sva package for removing batch effects and other unwanted variation in high-throughput experiments. Bioinformatics 2012; 28: 882-883.

39. Ritchie ME, Phipson B, Wu D, et al: limma powers differential expression analyses for RNAsequencing and microarray studies. Nucleic Acids Res. 2015; 43: e47-e47. Available at: https://doi.org/10.1093/nar/gkv007.

40. Kuo P-L, Wang S-T, Lin Y-M, et al: Expression profiles of the DAZ gene family in human testis with and without spermatogenic failure. Fertil. Steril. 2004; 81: 1034-1040.

41. Lin YM, Kuo PL, Lin YH, et al: Messenger RNA transcripts of the meiotic regulator BOULE in the testis of azoospermic men and their application in predicting the success of sperm retrieval. Hum. Reprod. 2005; 20: 782-788.

42. Bonaparte E, Moretti M, Colpi GM, et al: ESX1 gene expression as a robust marker of residual spermatogenesis in azoospermic men. Hum. Reprod. 2010; 25: 1398-1403.

43. Balhorn R: The protamine family of sperm nuclear proteins. Genome Biol. 2007; 8: 227. Available at: https://doi.org/10.1186/gb-2007-8-9-227.

44. Naranjo-Suarez S, Carlson BA, Tobe R, et al: Regulation of HIF-1a activity by overexpression of thioredoxin is independent of thioredoxin reductase status. Mol. Cells 2013; 36: 151-157.

\section{Tables}

Due to technical limitations, table 1-5 is only available as a download in the Supplemental Files section.

\section{Figures}
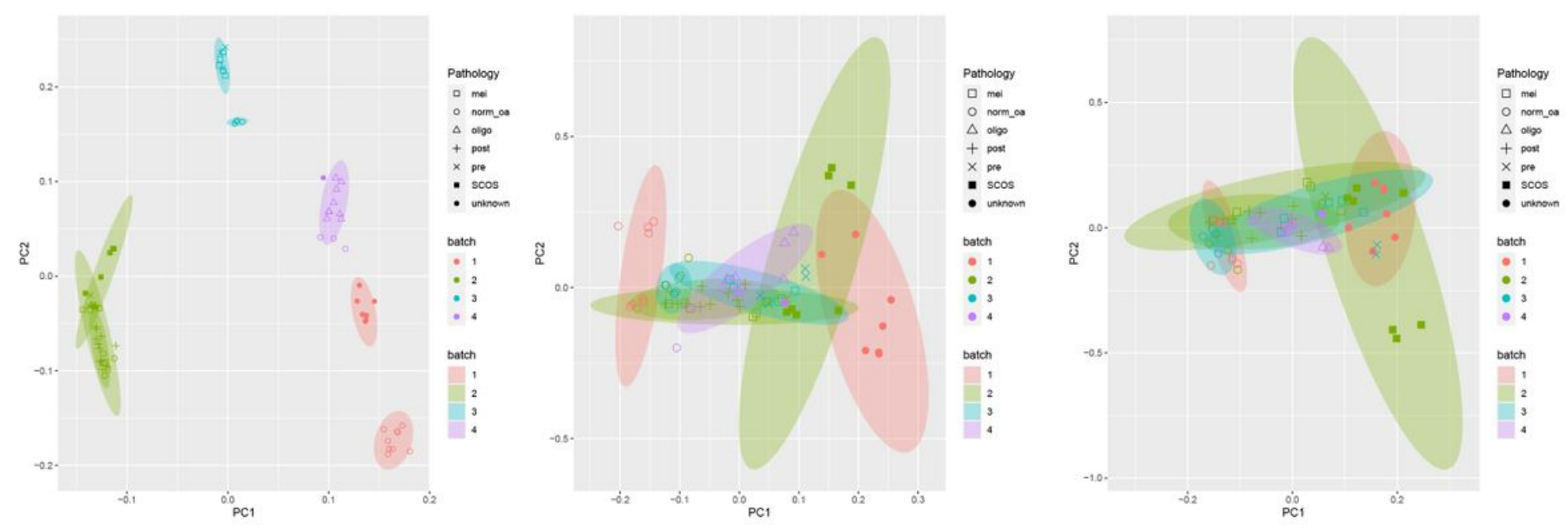

\section{Figure 1}

PCA of pooled samples before and after the batch effect removal using different algorithms. A) Before the batch effect removal, sample with identical or similar pathology were separated based on their batches. After the removal, PCA could separate sample according to their pathology and the samples 
were grouped regardless of their batches using limma algorithm (B) and SVA algorithm (C). Batch 1-4 represents GSE145467, GSE45885, GSE108886, GSE14310. Aberrations: mei (meiotic arrest); norm_oa (normal spermatogenesis or obstructive azoospermia); oligo (oligospermia); post (post meiotic arrest); pre (pre meiotic arrest); SCOS (Sertoli cell-only syndrome); unknown (azoospermia with unknown pathology).

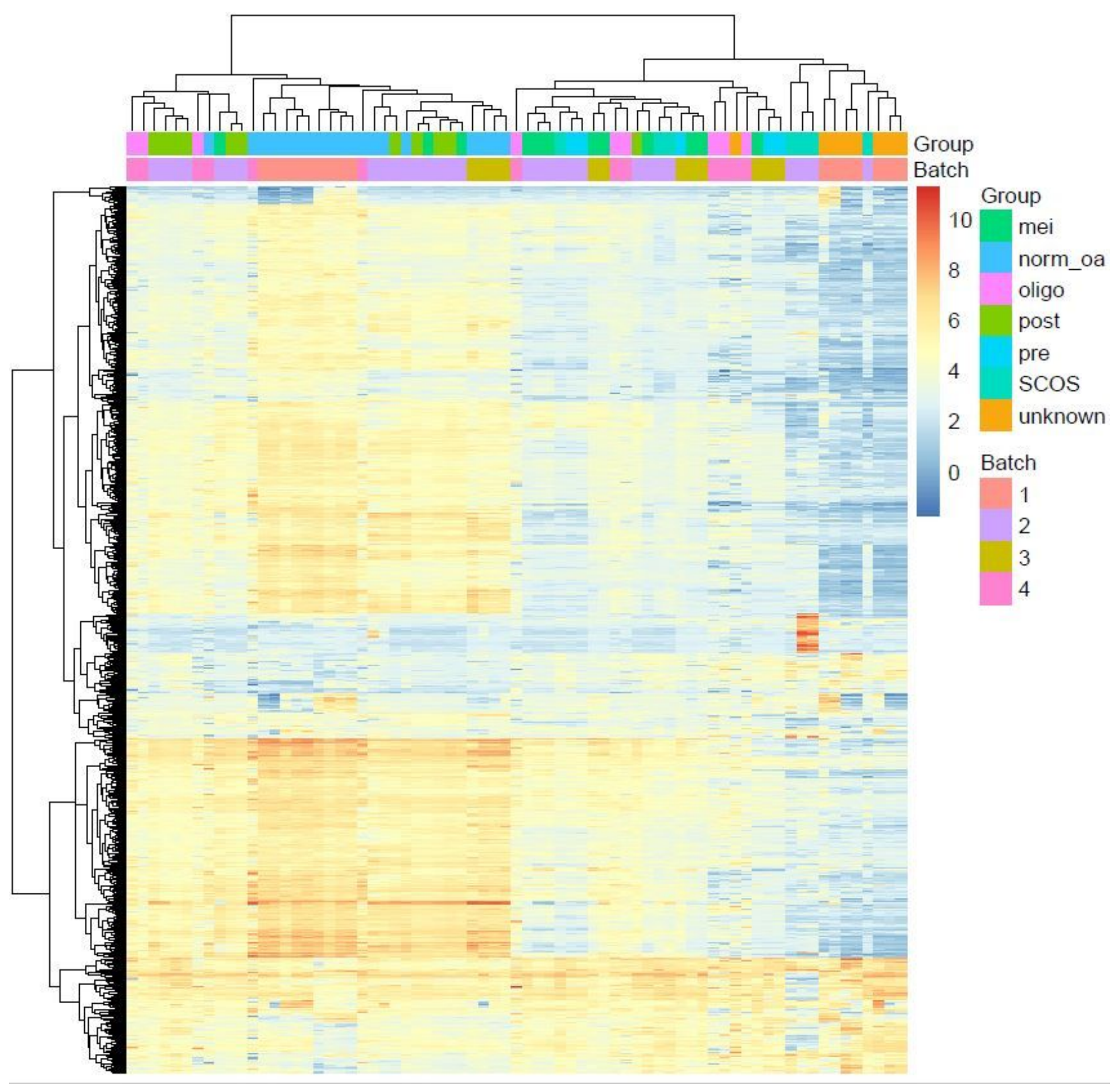

Figure 2 
A heatmap representing 71 samples, clustered based on correlation coefficient of 788 genes with standard deviation greater than 1. Group indicates the pathology of samples and the batch represents different datasets. Batch effect removal was approved as the heatmap clusters genes based on their pathologic groups and separates them based on their batches. Batch 1-4 represents GSE145467, GSE45885, GSE108886, GSE14310. Aberrations: mei (meiotic arrest); norm_oa (normal spermatogenesis or obstructive azoospermia); oligo (oligospermia); post (post meiotic arrest); pre (pre meiotic arrest); SCOS (Sertoli cellonly syndrome); unknown (azoospermia with unknown pathology).
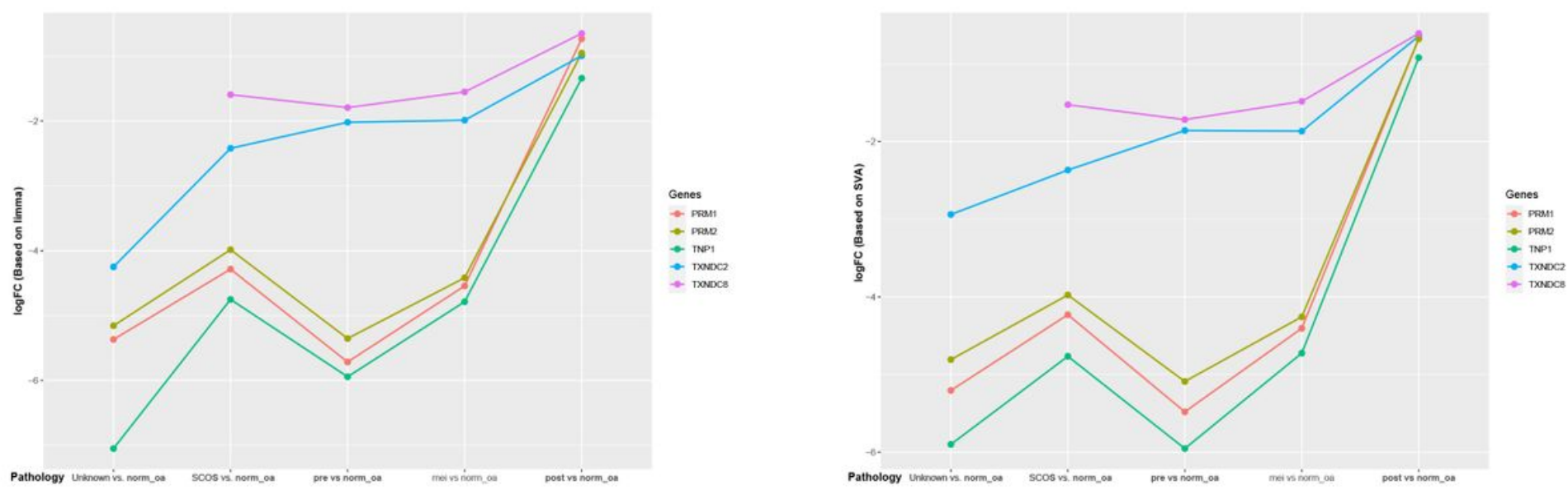

\section{Figure 3}

Log fold changes of TXNDC2, PRM1, PRM2 and TNP1 genes in different pathologies were illustrated. A) After the batch effect removal using limma package, different logFC of individual genes was visualized in different aberrant pathologies. B) A same pattern of logFC differences were also observed after using SVA for batch effect removal. In all comparisons, normal spermatogenesis was used as control.

Aberrations: mei (meiotic arrest); norm_oa (normal spermatogenesis or obstructive azoospermia); oligo (oligospermia); post (post meiotic arrest); pre (pre meiotic arrest); SCOS (Sertoli cell-only syndrome); unknown (azoospermia with unknown pathology); TXNDC2 (Thioredoxin Domain Containing 2); TXNDC8 (Thioredoxin Domain Containing 8); PRM1 (Protamine 1); PRM2 (Protamine 2); TNP1 (Transition Protein 1). 


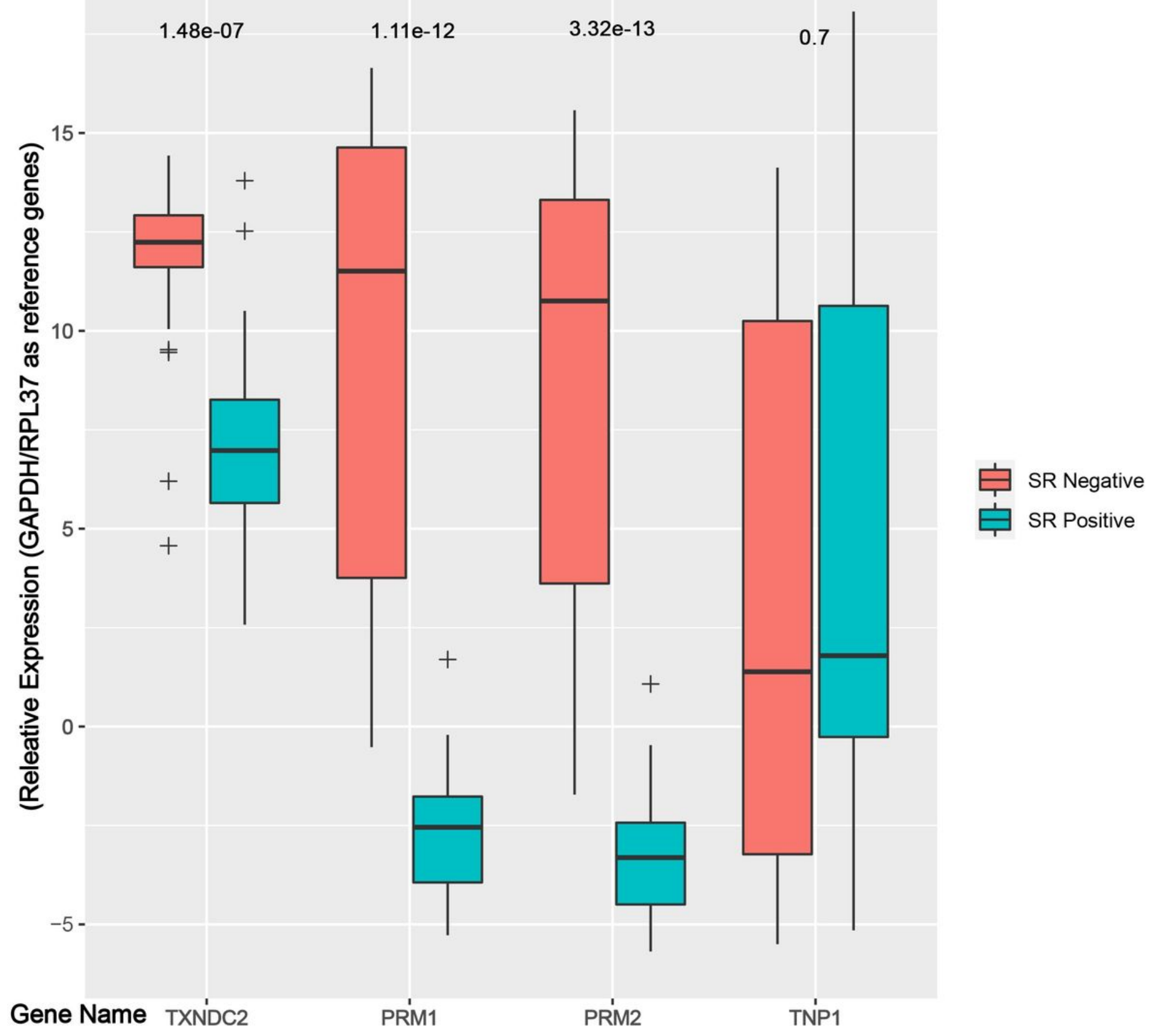

\section{Figure 4}

Relative expression of TXNDC2 and protamination genes were compared between men with positive (blue bars) and negative (red bars) sperm retrieval. Mean Cqs of both reference genes, GAPDH and RPL37, were calculated and used for relative expression. Meaningful intra-gene differences were illustrated for TXNDC2, PRM1 and PRM2. TNP1 showed overlapped relative expression between SR positive and negative samples. p-value less than 0.05 were considered as significant. 


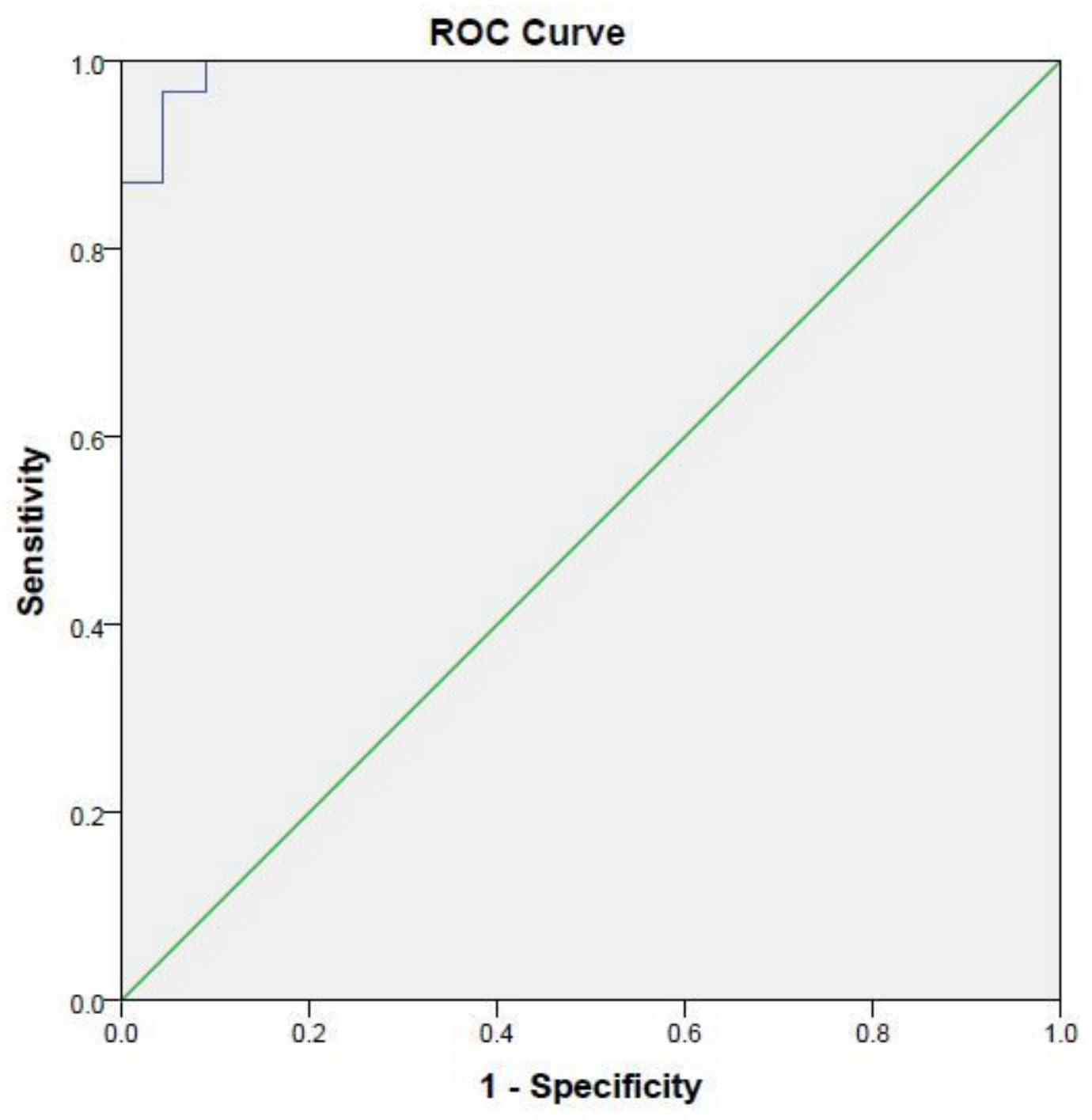

Figure 5

ROC analysis based on multiple regression model analysis of TXNDC2, PRM1, and PRM2. The area under the curve (AUC) or ROC value was determined as 0.993 (SE=0.0075, 95\% Cl: 0.978-1.000). the sensitivity and specificity was $96.8 \%$ and $95.5 \%$ respectively.

\section{Supplementary Files}

This is a list of supplementary files associated with this preprint. Click to download.

- SuppData.pdf

- table1.pdf

- table2.pdf 
- table3.pdf

- table4.pdf

- table5.pdf

Page $17 / 17$ 